\title{
DIGITAL STRAIGHT LINES IN THE KHALIMSKY PLANE
}

\author{
ERIK MELIN
}

\begin{abstract}
We consider the digital plane of integer points equipped with the Khalimsky topology. We suggest a digitization of straight lines such that the digitized image is homeomorphic to the Khalimsky line and a digitized line segment is a Khalimsky arc. It is demonstrated that a Khalimsky arc is the digitization of a straight line segment if and only if it satisfies a generalized version of the chord property introduced by Rosenfeld.
\end{abstract}

\section{Digitization of straight lines}

We are interested in representing lines in the digital plane, $Z^{2}$. One widely used digitization is the one considered by Azriel Rosenfeld [6]. Define the set

$$
\begin{aligned}
C(0)=\left\{x ; x_{1}=0 \text { and }-1 / 2<\right. & \left.x_{2} \leq 1 / 2\right\} \\
& \cup\left\{x ;-1 / 2<x_{1} \leq 1 / 2 \text { and } x_{2}=0\right\} .
\end{aligned}
$$

For each $p \in \mathrm{Z}^{2}$, let $C(p)=C(0)+p$. Note that $C(p)$ is a cross with center at $p$, that $C(p) \cap C(q)$ is empty if $p \neq q$, and that $\bigcup_{p \in Z^{2}} C(p)$ is equal to the grid lines $(\mathrm{R} \times \mathbf{Z}) \cup(\mathbf{Z} \times \mathbf{R})$. We now define the Rosenfeld digitization of $A \subset \mathrm{R}^{2}:$

$$
\mathscr{D}_{\mathrm{R}}: \mathscr{P}\left(\mathrm{R}^{2}\right) \rightarrow \mathscr{P}\left(Z^{2}\right), \quad \mathscr{D}_{\mathrm{R}}(A)=\left\{p \in \mathrm{Z}^{2} ; C(p) \cap A \neq \emptyset\right\} .
$$

Since the union of the crosses is the grid lines, a straight line or a sufficiently long line segment has non-empty digitization. We briefly discuss some terminology in this context: $C(p)$ is called a cell with nucleus $p$. In general, $C(p)$ may be any subset of a space $X$, and is defined for every $p$ in some subspace $Z$ of $X$. Using the definition above, the digitization of a subset of $X$ is a subset of $Z$, completely determined by the cells. If $(X, d)$ is a metric space and

$$
C(p) \subset\{x \in X ; \forall b \in Z, d(x, p) \leq d(x, b)\},
$$

Received September 6, 2003. 
then we talk of a Voronoi digitization. Such a digitization may be thought of as a reasonable metric approximation. Note that $\mathscr{D}_{\mathrm{R}}$ is a Voronoi digitization; $C(p)$ is contained in the set $\left\{x \in \mathrm{R}^{2} ;\|x-p\|_{\infty} \leq \frac{1}{2}\right\}$.

In the real plane, the concept of straight lines is old and very well known; a straight line is a set of the form $\{(1-t) a+t b ; t \in \mathrm{R}\}$, where $a$ and $b$ are two distinct points in the plane. A straight line segment is a connected subset of a line. We shall consider closed segments of finite length (possibly empty), which, unless empty, we may write as $\{(1-t) a+t b ; t \in[0,1]\}$, where $a$ and $b$ are the endpoints. We will denote such a segment by $[a, b]$. Like Rosenfeld, we will have to exclude lines and straight line segments with a slope $45^{\circ}+n \cdot 90^{\circ}$, where $n$ is an integer - for certain such lines, the Rosenfeld digitization is not an 8 -arc (see Section 5.1); to be precise, consider the line defined by $y=x+1 / 2$. The Rosenfeld digitization of this line is

$$
\{(n, n) ; n \in \mathrm{Z}\} \cup\{(n, n+1) ; n \in \mathbf{Z}\}
$$

and this set is too fat. This may seem unsatisfying, but fortunately this special case is trivial to handle if needed; we have to choose one of the terms of the union above.

In his famous paper [6], Rosenfeld used a slightly different digitization. For lines with slope strictly between $45^{\circ}$ and $-45^{\circ}$ he considered only the intersections with the vertical grid lines. Near the ends of line segments, this may result in a different digitization - namely if the line intersects a horizontal segment of a cross $C(p)$, and then ends before it reaches the vertical segment of the same cross. This, however, does not matter so much, since it only influences the length of the digital line segments; it does not affect the properties of the digitization. When we talk about the Rosenfeld digitization below, we mean our version, although it does not really matter which version we use. We will discuss some properties of the Rosenfeld digitization below, and motivate why we suggest another digitization. To begin with, we want to recall the definition of the Khalimsky topology.

\section{The Khalimsky topology}

There are different ways to introduce the Khalimsky topology on the integer line. One rather elegant way is to use a function $f: \mathbf{R} \rightarrow Z$ that approximates any real number by the closest integer (and when there is a choice, i.e., for the half-integers, always prefers the closest even integer). The Khalimsky topology on $\mathbf{Z}$ is the strongest topology such that $f$ is continuous, when $\mathbf{R}$ has the Euclidean topology. This definition has some immediate consequences. For example, since $Z$ is now the continuous image of a connected set, $Z$ is connected in the Khalimsky topology. 
We can also give the Khalimsky topology explicitly, by specifying a topological basis. For every even integer $m$, the set $\{m-1, m, m+1\}$ is open, and for every odd integer $n$ the singleton $\{n\}$ is open. A basis is given by

$$
\{\{2 n+1\},\{2 n-1,2 n, 2 n+1\} ; n \in \mathbf{Z}\} .
$$

It follows that even points are closed. A digital interval $[a, b] \cap \mathbf{Z}$ with the subspace topology is called a Khalimsky interval, and a homeomorphic image of a Khalimsky interval into a topological space is called a Khalimsky arc.

On the digital plane $Z^{2}$, the Khalimsky topology is given by the product topology. A point with both coordinates odd is open. If both coordinates are even, the point is closed. These types of points are called pure. Points with one even and one odd coordinate are neither open nor closed; these are called mixed.

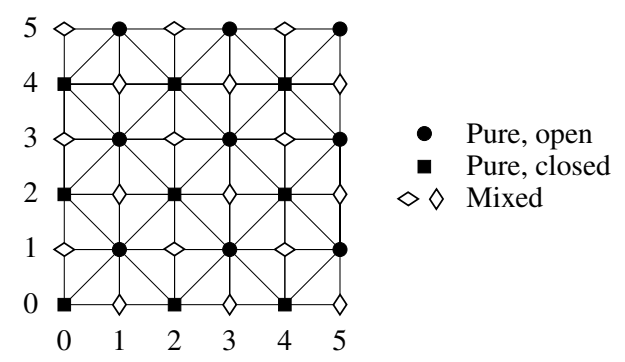

FIgURE 1. Part of the Khalimsky plane

In Figure 1, a part of the Khalimsky plane is shown; the lines indicate which points are connected. Note that the mixed points are only connected to their 4neighbors, whereas the pure points are connected to all eight neighbors. These properties are discussed in [2], [1], where also a more systematic study of the Khalimsky topology can be found.

\subsection{Continuous functions}

Having equipped $Z$ with the Khalimsky topology, we may speak of continuous functions $\mathbf{Z} \rightarrow \mathbf{Z}$. It is not hard to see that a continuous function $f$ must be Lipschitz with Lipschitz constant 1; we say it is Lip-1. Indeed, a set $\{m, n\}$ is connected if and only if $|m-n| \leq 1$. A continuous image of a connected set is connected; this means in particular that $|f(n+1)-f(n)| \leq 1$ for every $n$. Lip1 is however not sufficient for continuity. It is not hard to prove that $f: \mathbf{Z} \rightarrow \mathbf{Z}$ is continuous if and only if: (i) $f$ is Lip-1 and (ii) For every $x, f(x) \not \equiv x(\bmod 2)$ implies $f(x \pm 1)=f(x)$. (See for example Lemma 2.7 of [5].) Using this result, it is also easy to check that the mapping $\mathbf{Z} \rightarrow \mathbf{Z}^{2}, x \mapsto(x, f(x))$ is in fact a homeomorphism from $Z$ to its image, assuming $f$ to be continuous. 
Hence, the graph of a continuous function on a Khalimsky interval is always a Khalimsky arc.

\section{Notions of mathematical morphology}

We will need some concepts from mathematical morphology, see [8, Chapter II] or [4], for an introduction to this topic.

Let $(G,+)$ be a commutative group. We may then speak of Minkowski addition of two subsets;

$$
A+B=\{x+y ; x \in A, y \in B\}
$$

is called the Minkowski sum of $A$ and $B$. Given any subset $A$ of $G$ we also define the opposite set as $\check{A}=\{-x ; x \in A\}$. Let us fix a set $B \subset G$. The operation on subsets of $G$ defined by $A \mapsto A+B$ is called dilation by $B$. The operation $A \mapsto A \ominus B$, where $A \ominus B=\left(A^{c}+B\right)^{c}$ by definition, is called erosion by $B$. Combining these operations, we may define the $B$-opening as $A_{B}=(A \ominus \check{B})+B$, and the $B$-closure as $A^{B}=(A+\check{B}) \ominus B=\left((A+\check{B})^{c}+B\right)^{c}$. In this paper, we will only need to consider the $B$-closure. It can be shown that the mapping $A \mapsto A^{B}$ is extensive, increasing and idempotent under the order of set inclusion.

\section{Continuous digitization}

One drawback with the Rosenfeld digitization of $\mathbf{R}^{2}$ when one has equipped $Z^{2}$ with the Khalimsky topology is that the Rosenfeld digitization is designed to work well with 8-connectedness (see Section 5.1). In the Khalimsky plane, only pure points are connected to all 8 neighbors. In particular, this means that the Rosenfeld digitization of a line or straight line segment is not in general connected in the Khalimsky sense.

In this section, we will suggest an alternative digitization of a straight line. We will call this digitization the Khalimsky continuous digitization, or often just continuous digitization. The digitized line will be connected in the Khalimsky sense; in fact, it will be a homeomorphic image of the Khalimsky line. Let

$$
D(0)=\left\{(t, t) \in \mathrm{R}^{2} ;-1 / 2<t \leq 1 / 2\right\} \cup\left\{(t,-t) \in \mathrm{R}^{2} ;-1 / 2<t \leq 1 / 2\right\} .
$$

For each pure point $p \in \mathrm{Z}^{2}$, define $D(p)=D(0)+p$. Note that $D(p)$ is a cross, rotated $45^{\circ}$, with center at $p$, and that $D(p)$ is contained in the set $\left\{x \in \mathrm{R}^{2} ;\|x-p\|_{\infty} \leq 1 / 2\right\}$. This means that a digitization with $D(p)$ as a cell with nucleus $p$ is a Voronoi digitization. Compare with the $C(p)$ used in the Rosenfeld digitization discussed above. Let us first define an intermediate, 
pure digitization of a subset $A$ of $\mathbf{R}^{2}$ :

$$
\mathscr{D}_{\mathrm{P}}(A)=\left\{p \in \mathrm{Z}^{2} ; p \text { is pure and } D(p) \cap A \neq \emptyset\right\} .
$$

This digitization is the basis for continuous digitization. To make everything work as we wish, certain mixed points have to be added. In words, a point belongs to the continuous digitization if it belongs to the pure digitization or if the two points $\left(p_{1} \pm 1, p_{2}\right)$ or the two points $\left(p_{1}, p_{2} \pm 1\right)$ belong to the pure digitization. If $p$ is mixed, then $\left(p_{1}+1, p_{2}\right)$ and $\left(p_{1}-1, p_{2}\right)$ are both pure, and the operator adds the missing point $p$ in between, gluing these points together. Now we formally give the definition of continuous digitization:

Definition 4.1. Let $L$ be a Euclidean line or a line segment. The continuous digitization $\mathscr{D}(L)$ of $L$ is defined as follows: If $L$ is horizontal or vertical $\mathscr{D}(L)=\mathscr{D}_{\mathrm{R}}(L)$. Otherwise define $\mathscr{D}_{\mathrm{M}}(L)$ as

$\mathscr{D}_{\mathrm{M}}(L)=\left\{p \in \mathrm{Z}^{2} ;\left(p_{1} \pm 1, p_{2}\right) \in \mathscr{D}_{\mathrm{P}}(L)\right\} \cup\left\{p \in \mathrm{Z}^{2} ;\left(p_{1}, p_{2} \pm 1\right) \in \mathscr{D}_{\mathrm{P}}(L)\right\}$

and let

$$
\mathscr{D}(L)=\mathscr{D}_{\mathrm{P}}(L) \cup \mathscr{D}_{\mathrm{M}}(L) .
$$

As was the case for the Rosenfeld digitization, we will have to make an exception for a certain family of lines and line segments. Here it is the horizontal lines and the vertical lines; in the Rosenfeld case, it was the diagonal lines. In fact, the reason is precisely the same - the digitization of some lines would be too fat. As will be seen below, the first step of continuous digitization is closely related to Rosenfeld digitization, one difference being that the plane and the grid lines are rotated $45^{\circ}$.

Using notation from mathematical morphology, we can much simplify the formula in the definition above when $L$ is not horizontal or vertical. Compared with the pure digitization, certain mixed points are added in the continuous digitization. This process can be described as a morphological closure operation with the following set: $M=\{(0,0),(1,0),(0,1),(1,1)\}$. We have

$$
\mathscr{D}(L)=\left(\mathscr{D}_{\mathrm{P}}(L)\right)^{M}=\left(\left(\mathscr{D}_{\mathrm{P}}(L)+\check{M}\right)^{c}+M\right)^{c} .
$$

REMARK 4.2. While the morphological formula is a convenient way of writing the relatively complicated definition, an algorithm should probably instead be based on the following recursive approach (here formulated for lines with slope strictly between $0^{\circ}$ and $45^{\circ}$ ): Suppose that a pure point $p_{i}$ is in the digitization. If $L$ meets $D\left(p_{i}+(1,1)\right)$, let $p_{i+1}=p_{i}+(1,1)$, otherwise let $p_{i+1}=p_{i}+(1,0)$ and $p_{i+2}=p_{i}+(2,0)$. 

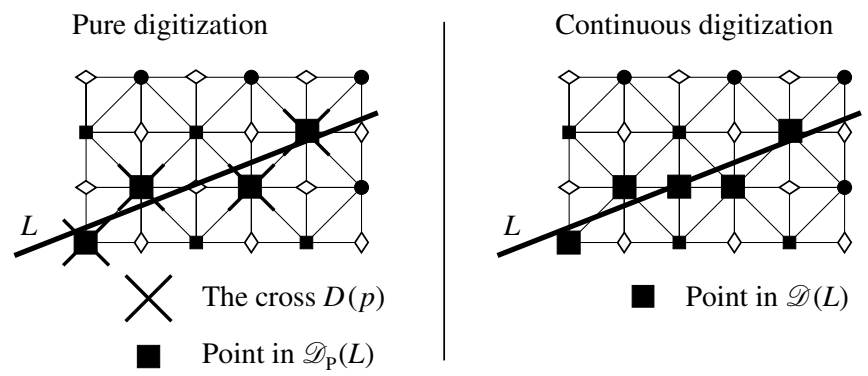

FIGURE 2. Example of pure and continuous digitization of a line segment

In Figure 2, this definition is illustrated. We will first show the approximation properties of this digitization are not too bad; to be precise, we will show that the distance from this digitization to the original line is not too big. To formulate it, we need consider the distance between a subset $A$ of a metric space $(X, d)$ and a point $x$ in the space defined by $d(A, x)=\inf _{y \in A} d(x, y)$. Sometimes, we will let $d^{p}$ denote the $l^{p}$-metric (where $1 \leq p \leq \infty$ ), in order to avoid misunderstandings. We also let $d^{p}$ denote the corresponding set-point distance.

REMARK 4.3. Let $L$ be a Euclidean line. It is easy to see that if $p$ is pure and $d^{\infty}(L, p)<1 / 2$, then $p \in \mathscr{D}(L)$. In contrast to this, note that the line may pass through a mixed point that does not belong to the digitization. For example, the line $y=x-1$ has the digitization $\{(n, n) ; n \in \mathbf{Z}\}$.

Proposition 4.4. Let $L$ be a Euclidean line in $\mathrm{R}^{2}$. Then $d^{\infty}(L, p) \leq 1 / 2$ for every $p \in \mathscr{D}(L)$. Moreover, for every $x \in L$ we have $d^{1}(\mathscr{D}(L), x) \leq 1$.

PRoof. For a horizontal, vertical or diagonal line, these statements are easy, so we need not consider such lines any further. For the first statement, suppose that $p \in \mathscr{D}(L)$. If $p$ is pure, then the statement is true by construction. If $p$ is mixed, then either $\left(p_{1} \pm 1, p_{2}\right) \in \mathscr{D}(L)$ or $\left(p_{1}, p_{2} \pm 1\right) \in \mathscr{D}(L)$. In the first case, $L$ meets both $D\left(p_{1}-1, p_{2}\right)$ and $D\left(p_{1}+1, p_{2}\right)$, and therefore must pass through $\left\{y \in \mathrm{R}^{2} ;\|y-p\|_{\infty} \leq 1 / 2\right\}$. The other case is similar.

To prove the second statement, note that the family of sets

$$
\left\{\left\{x \in \mathbf{R}^{2} ; d^{1}(x, p) \leq 1\right\} ; p \text { is pure }\right\}
$$

covers the plane. Let $x \in L$ and $p$ be a pure point such that $d^{1}(x, p) \leq 1$. If $p \in \mathscr{D}(L)$ we are done, so suppose this is not so. Then $d^{\infty}(x, p) \geq 1 / 2$, which means that $x$ belongs to one of the four triangles in the set

$$
\left\{x \in \mathrm{R}^{2} ; d^{1}(x, p) \leq 1 \text { and } d^{\infty}(x, p) \geq 1 / 2\right\} .
$$


Suppose for definiteness that $x$ belongs to the right-most triangle; in other words that $x_{1} \geq p_{1}+1 / 2$. By combining the slope assumption with the fact that $L$ does not meet $D(p)$, we conclude that $L$ intersects $D(p+(1,1))$ and $D(p+(1,-1))$. But this implies that $p+(1,0) \in \mathscr{D}(L)$ and clearly $d^{1}(x, p+(1,0)) \leq 1$.

Now we turn to the theorem which motivates the definition of continuous digitization:

THEOREM 4.5. Suppose that $L$ is a real line. Then $\mathscr{D}(L)$ is homeomorphic to the Khalimsky line. If $[a, b]$ is a line segment, then $\mathscr{D}([a, b])$ is a Khalimsky arc (possibly empty).

Proof. For a horizontal, vertical or diagonal line or line segment, the conclusion follows easily. Let $L$ be a real line. We may restrict ourselves to the case where the slope is strictly between 0 and $45^{\circ}$ - this is sufficient because of symmetry in the definition. First we show that there are no two points $x$ and $y$ in the digitization such that $x_{1}=y_{1}$ and $x_{2} \neq y_{2}$. We consider two cases. If $x$ is mixed, then by the slope assumption, the two pure points $\left(x_{1} \pm 1, x_{2}\right)$ belong to the digitization. Therefore $L$ cannot meet any $D(p)$ of a pure point with the same first coordinate as $x$. Nor can it meet any $D\left(p_{1}, p_{2}\right)$ where $p_{1}=x_{1}-1$ and $p_{2} \geq x_{2}+2$ or where $p_{1}=x_{1}+1$ and $p_{2} \leq x_{2}-2$. Hence there cannot be any other mixed point in the digitization with the same first coordinate.

If $x$ is pure, then $L$ cannot meet any $D(y)$ of a pure point with $y_{1}=x_{1}$ and $y_{2} \geq x_{2}+2$ or $y_{2} \leq x_{2}-2$. Nor can it meet any $D\left(p_{1}, p_{2}\right)$ where $p_{1}=x_{1}-1$ and $p_{2} \geq x_{2}+1$ or where $p_{1}=x_{1}+1$ and $p_{2} \leq x_{2}-1$, and as before, there cannot be any mixed point with the same first coordinate.

The argument above shows that $\mathscr{D}(L)$ can be written as the graph of a function $f: \mathbf{Z} \rightarrow \mathbf{Z}$. Now we show that $f$ is in fact continuous, and hence that the map $\mathbf{Z} \rightarrow \mathscr{D}(L), n \mapsto(n, f(n))$ is a homeomorphism.

Suppose $x=(n, f(n)) \in \mathscr{D}(L)$ is mixed, so that $f(n)$ and $n$ have different parity. Then $\left(x_{1} \pm 1, x_{2}\right)$ belong to $\mathscr{D}(L)$ by definition, which means that $f(n \pm 1)=f(n)$ as required.

If $x=(n, f(n)) \in \mathscr{D}(L)$ is pure, so that $f(n)$ and $n$ agree in parity, then $L$ may meet either $D\left(x_{1}+1, x_{2}+1\right)$ so that $f(n+1)=f(n)+1$, or $L$ will intersect $D\left(x_{1}+2, x_{2}\right)$, which implies $f(n+1)=f(n)$. A similar argument shows that $f(n-1)=f(n)-1$ or $f(n-1)=f(n)$. Therefore $f$ is continuous. Finally, the digitization of a segment $[a, b]$ is clearly a connected, finite, subset of the digitization of the corresponding line, and therefore homeomorphic to a Khalimsky interval.

The following corollary of Theorem 4.5 and Proposition 4.4 shows that 
the continuous digitization is, in a certain metric sense, the best Khalimskycontinuous digitization possible.

Corollary 4.6. Suppose that $\varphi: \mathbf{Z} \rightarrow \varphi(\mathbf{Z}) \subset \mathbf{Z}^{2}$ is a homeomorphism, and that $L$ is a line in $\mathrm{R}^{2}$. Then

$$
\sup _{p \in \mathscr{D}(L)} d^{\infty}(L, p) \leq \sup _{p \in \varphi(\mathbf{Z})} d^{\infty}(L, p) .
$$

Proof. Note that if $\varphi(\mathbf{Z})$ contains precisely the same pure points as $\mathscr{D}(L)$, then $\varphi(\mathbf{Z})=\mathscr{D}(L)$ and since $\mathscr{D}(L)$ is the graph of a monotone function, the pure points in $\varphi(\mathbf{Z})$ cannot be a proper subset of the pure points in $\mathscr{D}(L)$. Therefore, if $\mathscr{D}(L) \neq \varphi(\mathbf{Z})$, there must be a pure point $q$ such that $q \in \varphi(\mathbf{Z})$ and $q \notin \mathscr{D}(L)$. But then

$$
\sup _{p \in \mathscr{D}(L)} d^{\infty}(L, p) \leq 1 / 2 \leq d^{\infty}(L, q) \leq \sup _{p \in \varphi(\mathrm{Z})} d^{\infty}(L, p)
$$

as required.

\section{Chord measures}

In this section we will present the chord property introduced by Rosenfeld [6] to characterize the sets that are the Rosenfeld digitization of line segments. Our goal is to give a generalized version that does the same with the continuous digitization.

The chord property will here be defined using a function we will call the chord measure. This will allow not only a characterization of which sets are digital lines, but also provide a qualitative measure; a smaller chord measure means a better approximation of a line. Assume that $R^{2}$ is equipped with a metric $d$. Denote by $\mathscr{F}^{2} \subset \mathscr{P}\left(Z^{2}\right)$ the family of finite subsets of the digital plane. Let $A \in \mathscr{F}^{2}$ be a finite set, and let $p$ and $q$ be points in $A$. Denote by $H$ the distance from the line segment $[p, q]$ to $A$ defined by:

$$
H: \mathscr{F}^{2} \times \mathrm{Z}^{2} \times \mathrm{Z}^{2} \rightarrow \mathrm{R}, \quad H(A, p, q)=\sup _{x \in[p, q]} \min _{m \in A} d(m, x) .
$$

REMARK 5.1. The distance function $H$ above, is related to the Hausdorff distance between subsets of a metric space $(X, d)$. The Hausdorff distance between two subsets $A, B \subset X$ is defined by:

$$
d(A, B)=\max \left(\sup _{y \in B} d(A, y) ; \sup _{x \in A} d(B, x)\right) .
$$

If $B=[p, q]$ is a line segment and $A$ a finite set as above, then clearly $H(A, p, q)=\sup _{y \in B} d(A, y)$, i.e., the first term in the Hausdorff distance. 
What about the other term? If we let $A$ be the Rosenfeld digitization of a relatively long line segment, and $p=q$ be one of the two endpoints of the digitized line. Then $B=[p, q]$ is a one-point set. Clearly $\sup _{x \in A} d(B, x)=$ $\sup _{x \in A} d(p, x)$ has a value that is comparable in magnitude with the length of the line segment. Below we will consider the maximum of $H(A, p, q)$ over all line segments $[p, q]$. A small value of this maximum will mean a good digitization. Since the second term of the Hausdorff distance has a maximum (taken again over the line segments) comparable with the length of the line segment, it is of little use in this context.

Definition 5.2. Let $A \in \mathscr{F}^{2}$ be a finite set. Then the chord measure of $A$, denoted by $\zeta(A)$, is defined by:

$$
\zeta(A)=\max _{p, q \in A} H(A, p, q)=\max _{p, q \in A} \sup _{x \in[p, q]} \min _{m \in A} d(m, x) .
$$

Definition 5.3. Let $A \in \mathscr{F}^{2}$. We say that $A$ has the chord property for the metric $d$ if $\zeta(A)<1$ for the metric $d$.

Rosenfeld did not use the chord measure. He defined this property directly, as in the following proposition.

Proposition 5.4. Let $A \in \mathscr{F}^{2}$ be a finite set. Then $A$ has the chord property for the $l^{\infty}$-metric if and only if for every pair $p, q$ of points in $A$, the line segment $[p, q] \subset A+B$, where $B=\left\{x \in \mathbf{R}^{2} ;\|x\|_{\infty}<1\right\}$ is the unit disk in the $l^{\infty}$ norm.

Proof. Suppose that $A$ has the chord property, so that $\zeta(A)<1$. Then it is immediately clear that the other statement holds. (In particular $\zeta(\emptyset)=-\infty$ and the statement is vacuously true for the empty set.) Conversely, suppose that $\zeta(A) \geq 1$. Then there are points $p$ and $q$ in $A$ such that $H(A, p, q) \geq 1$. But since $[p, q]$ is a compact set, and the $l^{\infty}$-metric is continuous for the Euclidean topology, there is an $x \in[p, q]$ such that $\min _{m \in A} d(x, m) \geq 1$. But then also the other statement does not hold.

\subsection{Chord measures and Rosenfeld digitization}

When discussing the Rosenfeld digitization, it is natural to consider $Z^{2}$ to be 8-connected, i.e., any of the horizontal, vertical or diagonal neighbors of a point is connected to the point. Given two points $x$ and $y$ we say that $x$ is adjacent to $y$ if and only if $\|x-y\|_{\infty}=1$. An 8-arc is a finite, connected, subset $A$ of $Z^{2}$, such that all but at most two points of $A$ have exactly two adjacency points, and the two exceptional points (the endpoints) have exactly one adjacency point in $A$. 
Rosenfeld [6] proved that the Rosenfeld digitization of a straight line segment is an 8 -arc with chord property for the $l^{\infty}$-metric and conversely, that if an 8 -arc has the chord property for the $l^{\infty}$-metric, then it is the digitization of a straight line segment.

\section{Chord measures and continuous digitization}

In this section we will use the chord measure introduced above to characterize the continuous digitization of a straight line segment. As with the Rosenfeld digitization, it is possible to show that a continuous digitization satisfies the chord property for a certain metric and, conversely, under some natural extra conditions, that a Khalimsky arc satisfying this chord property is the digitization of a straight line segment.

We will need to consider a somewhat special metric. Let $\delta(x, y)$ be the metric on $\mathrm{R}^{2}$ defined by $\delta(x, y)=\max \left(\left|x_{1}-y_{1}\right| / 2,\left|x_{2}-y_{2}\right|\right)$; in essence the $l^{\infty}$-metric rescaled in the first coordinate. In general, of course, we may for each positive $\alpha$ define a metric $\delta_{\alpha}(x, y)=\max \left(\alpha\left|x_{1}-y_{1}\right|,\left|x_{2}-y_{2}\right|\right)$. The following two examples show that $\alpha=1 / 2$ is not a random choice.

EXAMPLE 6.1. Let for each positive integer $L_{m}=[(0,0),(2 m+2,2 m)]$. Then

$\mathscr{D}\left(L_{m}\right)=\{(n, n) ; 0 \leq n \leq m\} \cup\{(n+2, n) ; m \leq n \leq 2 m\} \cup\{(m+1, m)\}$.

Consider the segment $[(0,0),(m+2, m)]$, and in particular the point $r=$ $(m+1-2 / m, m-1)$ on this segment. The only point $p \in \mathscr{D}\left(L_{m}\right)$ with $\left|p_{2}-r_{2}\right|<1$ is $p=(m-1, m-1)$. We have $\left|p_{1}-r_{1}\right|=2-2 / m$, but for a fixed $\alpha>1 / 2$, we can choose an $m$ such that $\alpha\left|p_{1}-r_{1}\right|>1$. Therefore $\mathscr{D}\left(L_{m}\right)$ does not have the chord property for the $\delta_{\alpha}$-metric.

EXAMPLE 6.2. It is easy to check that the Khalimsky arc

$$
D=\{(0,0),(1,0),(2,0),(3,0),(4,0),(5,1),(6,2)\}
$$

is not the continuous digitization of a straight line segment. (Use Theorem 6.3 below.) Note that the critical point is the point $(3,1)$ on the segment $[(0,0)$, $(6,2)$ ]. If however $\alpha<1 / 2$, then $D$ will have the chord property for the $\delta_{\alpha}$-metric.

A striking property of the $\delta$-metric is that it is not invariant under permutation of the coordinates. A consequence is that we have to consider two separate cases: With the definition above, we will handle lines with slope between $-45^{\circ}$ and $45^{\circ}$ and Khalimsky arcs that are graphs of a function of the first variable, i.e., $\left\{(x, f(x)) \in \mathbf{Z}^{2} ; x \in I \subset \mathrm{Z}\right\}$. In the other case, we first have 
to permute the coordinates, so that the criteria above is fulfilled. Note that the digitization of a straight line segment is always the graph of a continuous function in some variable.

In order to avoid complicated notation or a complicated formulation of the theorems below, we will define the $\delta^{1}$-metric to be the $\delta$-metric above, and the $\delta^{2}$-metric to be the same metric after permutation of the coordinates; in other words, the $l^{\infty}$-metric rescaled in the second coordinate. With the term some $\delta$-metric we mean one of these metrics.

THeOREM 6.3. The continuous digitization of a straight line segment is a Khalimsky arc (possibly empty) having the chord property for some $\delta$-metric.

Proof. Let $D$ be the continuous digitization of a line segment $L$. Theorem 4.5 shows that $D$ is a Khalimsky arc. When $L$ is a diagonal or horizontal line segment, the second statement is easy, so we need not consider these cases any further. Assume therefore that the slope of $L$ is strictly between $0^{\circ}$ and $45^{\circ}$. The case with slopes between $0^{\circ}$ and $-45^{\circ}$ is similar.

Suppose now that $p, q \in D$ and let $r \in[p, q]$. It follows from Proposition 4.4 that there is a point $s \in L$ such that $d^{\infty}(s, r) \leq \frac{1}{2}$. We have to prove that there is a point $a \in D$ such that $\delta(a, r)<1$. Consider first the case $r$ is a pure point. Unless $s=\left(r_{1}-\frac{1}{2}, r_{2}+\frac{1}{2}\right)$, it follows immediately that $r \in D$. (If $s=\left(r_{1}-\frac{1}{2}, r_{2}-\frac{1}{2}\right)$ we may use the assumption on the slope to conclude that $r \in D$.) Suppose $s=\left(r_{1}-\frac{1}{2}, r_{2}+\frac{1}{2}\right)$. Then no pure point in $D$, and therefore no point in $D$, can be on or below the line through $r$, parallel with $L$. This contradicts the fact that $r \in[p, q]$.

Next suppose that $r_{2} \in \mathbf{Z}$, but $r$ is not pure. Then there is a pure point $m$ such that $m_{2}=r_{2}$ and $\left.r \in\right] m, m+(2,0)$ [. Clearly $m_{1}-\frac{1}{2}<s_{1}<m_{1}+\frac{5}{2}$ and $\left|m_{2}-s_{2}\right| \leq \frac{1}{2}$. Suppose that $m \notin D$. Then $L$ must intersect the segment $\left[\left(m_{1}+\frac{1}{2}, m_{2}-\frac{1}{2}\right),\left(m_{1}+\frac{5}{2}, m_{2}-\frac{1}{2}\right)[\right.$, and it follows that $m+(2,0) \in D$. Since $\left|m_{1}-r_{1}\right|<2,\left|m_{1}+2-r_{1}\right|<2$ and $m_{2}=r_{2}$, both $m$ and $m+(2,0)$ have a $\delta$-distance strictly less than one from $r$, and at least one of them is in $D$.

Finally, we study the case $r_{2} \notin Z$. Note that if $s$ is in the parallelogram $\left\{x \in \mathbf{R}^{2} ; m_{2}-1 / 2<x_{2} \leq m_{2}+1 / 2\right.$ and $\left.m_{2}-m_{1}-1 \leq x_{2}-x_{1}<m_{2}-m_{1}+1\right\}$, where $m$ is pure, it follows that $m \in D$, that $\left|m_{1}-s_{1}\right|<\frac{3}{2}$ and that $\left|m_{2}-s_{2}\right| \leq$ $\frac{1}{2}$. Since these parallelograms cover $\mathbf{R}^{2}$, there is an $m$ such that $s$ belongs to the corresponding parallelogram. From the triangle inequality, it follows that $\left|m_{1}-r_{1}\right| \leq\left|m_{1}-s_{1}\right|+\left|s_{1}-r_{1}\right|<2$ and similarly that $\left|m_{2}-r_{2}\right| \leq 1$. But since we have excluded the case $r_{2} \in Z$, the last inequality is also sharp. Hence $\delta(m, r)<1$ and the theorem is proved. 
The converse of this theorem is formulated below. The proof is an adaptation of Kiselman's [4, Theorem 10.4] proof of Rosenfeld's corresponding theorem. Rosenfeld's original proof can also be adapted to work, however, the present proof is much shorter.

THEOREM 6.4. Suppose that a Khalimsky arc $D=\{(x, f(x)) ; x \in I\} \subset \mathrm{Z}^{2}$ is the graph of a monotone, continuous function $f$, and that $D$ has pure endpoints. If $D$ has the chord property for the $\delta^{1}$-metric, then $D$ is the continuous digitization of a line segment.

Proof. Let $D$ be the graph of a, say, increasing and continuous function $f$, and let $D$ have the chord property for the $\delta^{1}$-metric. Given three real numbers $\alpha, \beta, \rho$ we define a strip in the plane by

$$
S(\alpha, \beta, \rho)=\left\{x \in \mathrm{R}^{2} ; \alpha x_{1}+\beta-\rho(1+\alpha) \leq x_{2} \leq \alpha x_{1}+\beta+\rho(1+\alpha)\right\} .
$$

Let us call the number $\rho$ the diagonal width of the strip. The boundary, $\partial S(\alpha, \beta, \rho)$, of the strip consists of two components given by the lines $x_{2}=$ $\alpha x_{1}+\beta \pm \rho(1+\alpha)$, i.e., the center line, $x_{2}=\alpha x_{1}+\beta$, translated by the vectors $(-\rho, \rho)$ and $(\rho,-\rho)$. Observe that a set of pure points is a subset of the digitization of a straight line segment if and only if it is contained in a strip with a diagonal width strictly less than $\frac{1}{2}$.

For a given $\alpha$ there is a smallest strip $S(\alpha, \beta, \rho)$ containing $D$. If we also allow $\alpha$ to vary, there is a strip $S_{0}=S\left(\alpha_{0}, \beta_{0}, \rho_{0}\right)$ of smallest diagonal width. If $D$ consists of only one or two pure points, the conclusion follows easily, so we may assume that $D$ consist of at least three pure points. Clearly, there must be at least one point of $D$ in each component of the boundary of $D$; otherwise we could adjust $\beta$, and decrease $\rho$ to obtain a narrower strip. Also, one of these lines must contain a second point of $D$; otherwise we could rotate the lines slightly to obtain a strip of smaller diagonal width.

To demonstrate this, note that because of the assumption that $f$ is increasing, we cannot have two points $p$ and $q$ in $D$ such that $p=q+(t,-t)$ for some real $t$. Suppose that $q$ is the only point on the lower boundary and $p$ is the only point on the upper boundary. If $p$ is below the diagonal line $\{q+(t,-t) ; t \in \mathrm{R}\}$ we may rotate the upper line around $p$ and the lower line around $q$ clockwise, by decreasing $\alpha$, and adjusting $\beta$ and $\rho$, to get a narrower strip. If $p$ is above $\{q+(t,-t) ; t \in \mathrm{R}\}$, we should instead rotate by increasing $\alpha$.

In fact, we may assume that there are two points $p, q$ on one line, and a point $s$ on the other line, and the diagonal projection of $s$ onto the $p q$-line is on the open segment $] p, q[$. Otherwise we can again rotate the line somewhat to get a narrower strip.

For definiteness, we shall assume that the three points of the boundary are $p, s, q$, where $p$ and $q$ are on the lower boundary and $s$ on the upper boundary. 
Let $r=s+\left(2 \rho_{0},-2 \rho_{0}\right) \in[p, q]$ be the diagonal projection of $s$ onto $[p, q]$. If $D$ is not a subset of a digitization of a straight line, then $\rho_{0}$ is at least $\frac{1}{2}$. We will show that then there is no point $d \in D$, such that $\delta^{1}(d, r)<1$, or in other words that $D$ does then not satisfy the chord property for the $\delta^{1}$-metric. Now, since $2 \rho_{0} \geq 1$, such a point $d$ must satisfy $d_{2}<s_{2}$. As $D$ is the graph of an increasing function, we can therefore exclude any point with $d_{1} \geq s_{1}$. But again, since $2 \rho_{0} \geq 1$, it follows that $r_{1} \geq s_{1}+1$, so that also any $d$ with $d_{1} \leq s_{1}-1$ can be excluded. We conclude that there is indeed no $d \in D$ such that $\delta^{1}(d, r)<1$.

So far, we have proved that a Khalimsky arc $D$ with the chord property that is the graph of an increasing function is a subset of the digitization of some straight line $x_{2}=\alpha_{0} x_{1}+\beta_{0}$. Since $D$ is a Khalimsky arc with pure endpoints, it is clear that $D$ is the digitization of a compact, connected subset of the line, i.e., a line segment.

REMARK 6.5. The requirement that $D$ is the graph of a monotone function is necessary. Consider the set $K=\{(0,0),(1,1),(2,0)\}$. It is easy to see that $K$ is a Khalimsky arc with pure endpoints, that satisfies the chord property for the $\delta^{1}$-metric. However $K$ is not the digitization of a line segment.

Remark 6.6. An essential fact used in the proof is that if $\rho_{0}<\frac{1}{2}$, then $D$ is the digitization of the center line, $x_{2}=\alpha_{0} x_{1}+\beta_{0}$. This follows from Remark 4.3. Note that $D(p)$ is a rotated cross, and we use the diagonal width. In Kiselman's proof of Rosenfeld's theorem, the cross is not rotated, and he considers the vertical height of the strip.

\section{Concluding remarks}

In this section we will discuss some further properties of continuous digitization and compare it with Rosenfeld digitization. Let $f: Z \rightarrow Z$ be a continuous function, and let $G_{f}=\left\{(x, f(x)) \in \mathbf{Z}^{2} ; x \in \mathrm{Z}\right\}$ be its graph. It is straightforward to check that the complement of the graph consists of precisely two connectivity components, namely

$$
\left\{x \in \mathbf{Z}^{2} ; x_{2}<f\left(x_{1}\right)\right\} \quad \text { and } \quad\left\{x \in \mathbf{Z}^{2} ; x_{2}>f\left(x_{1}\right)\right\} .
$$

This implies that the continuous digitization of a line separates the Khalimsky plane into two components, precisely as a Euclidean line separates the Euclidean plane. This property does not hold in general for a Rosenfeld line if the plane is considered to be 8-connected, nor for the Khalimsky plane. However, the property does hold for the 4-connected plane and Rosenfeld lines. Compare with the Jordan curve theorem in a digital setting [7], [3]. 
Another consequence is that if two Euclidean lines cross, and thus has a common point, then the digitized lines also have at least one common point. To see this, observe that since the digitization of one line separates the plane, it also separates the other line. This property does not hold for the Rosenfeld lines.

Example 7.1. Let $L_{1}=\{(t, t) ; t \in \mathrm{R}\}$ and $L_{2}=\{(t,-t)+(0,1) ; t \in \mathrm{R}\}$ be two line in the plane. Then $\mathscr{D}_{\mathrm{R}}\left(L_{1}\right)=\{(n, n) ; n \in \mathrm{Z}\}$ and $\mathscr{D}_{\mathrm{R}}\left(L_{2}\right)=$ $\{(n,-n)+(0,1) ; n \in \mathbf{Z}\}$, so clearly $\mathscr{D}_{\mathrm{R}}\left(L_{1}\right) \cap \mathscr{D}_{\mathrm{R}}\left(L_{2}\right)$ is empty. This is the natural example, but there is one problem: The Rosenfeld digitization has an exception for these lines. However, if we rotate these lines just a little around the origin, the digitization near the origin will still be the same, say for every $p \in \mathrm{Z}^{2}$ with $\|p\|_{\infty}<10$. Now there is no exception, and the same conclusion holds.

AcKnowledgment. I am grateful to Christer Kiselman and Hania UsckaWehlou for helpful comments on earlier versions of this paper.

\section{REFERENCES}

1. Khalimsky, Efim, Topological structures in computer science, J. Appl. Math. Simulation Vol. 1 no. 1 (1987), 25-40.

2. Khalimsky, Efim, Kopperman, Ralph, Meyer, Paul R., Computer graphics and connected topologies on finite ordered sets, Topology Appl. 36 (1990), 1-17.

3. Kiselman, Christer O. Digital Jordan curve theorems in Discrete Geometry for Computer Imagery, 9th International Conference, DGCI 2000, Uppsala, Sweden, December 13-15 2000, (Eds. Gunilla Borgefors, Ingela Nyström, Gabriella Sanniti di Baja), Lecture Notes in Comput. Sci. 1953 (2000), 46-56.

4. Kiselman, Christer O. Digital geometry and mathematical morphology, Lecture Notes, Uppsala University, Department of Mathematics (2002), available at www.math.uu.se/ Kiselman

5. Melin, Erik, Connectedness and continuity in digital spaces with the Khalimsky topology, Project Report, Uppsala University, Department of Mathematics, 9 (2003), available at www.math.uu.se/ $/$ melin

6. Rosenfeld, Azriel, Digital straight line segments, IEEE Trans. Comput. c-23 No. 12 (1974), 1264-1269.

7. Rosenfeld, Azriel, Digital topology, Amer. Math. Monthly 86 (1979), 621-630.

8. Serra, Jean, Image Analysis and Mathematical Morphology, Academic Press (1982) xvii + $610 \mathrm{pp}$.

UPPSALA UNIVERSITY

DEPARTMENT OF MATHEMATICS

BOX 480, SE-751 06 UPPSALA

SWEDEN

E-mail:melin@math.uu.se 